\title{
N-Substituted Arylsulfonamides as Alternative Building Blocks in Peptoid and Peptide Synthesis
}

\author{
Simon Vézina-Dawod, Steve Jobin, Antoine Derson, Claire Herby, and \\ Eric Biron
}

Faculty of Pharmacy, Université Laval and Laboratory of Medicinal Chemistry, CHU de Québec Research Centre, CHUL, Quebec (QC), G1V 4G2, Canada

\section{Introduction}

Peptoids (oligo $N$-substituted glycines) are peptidomimetic oligomers showing attractive structural and pharmacological properties for the development of therapeutic candidates and molecular tools $[1,2]$. Compared to peptides, peptoids are resistant to proteases and show improved cell permeability $[3,4]$. Moreover, their synthesis is straightforward and the great chemical diversity that can be accessed with peptoids has prompted their use in combinatorial libraries. The submonomer method introduced by Zuckermann, et al. is the most frequently used approach to prepare peptoids [5]. This method is performed on solid support and involves iterative acylation and amination reactions to build up peptoid residues and generate oligomers after multiple rounds. A major advantage of this method is the large number of commercially available primary amines that allows the incorporation of an important chemical diversity. However, despite the great diversity of available primary amines, we observed that the number of submonomers bearing protected hydroxyl side chains is very limited. The very few that are commercially available are expensive. Protection of hydroxyl groups is recommended to avoid $O$-acylation during the acylation but the preparation of $O$-protected submonomers usually requires multistep synthesis or harsh conditions and they are recovered in low yields or often obtained as inconvenient gummy oils. To overcome these drawbacks and introduce interesting reactive or polar functionalities into peptoids, our strategy was to use amino alcohols as starting material and perform a two-step synthesis involving $N$-protection with an arylsulfonyl group followed by $O$-protection with tert-butyl to prepare fully protected submonomers. Then, the $N$-substituted arylsulfonamides could be used directly for the nucleophilic displacement step on solid support in the submonomer approach or for the synthesis of protected $N$-substituted glycines for the monomer approach (Figure 1).

\section{Results and Discussion}

The 2-nitrobenzenesulfonyl group (2-Nos) was particularly interesting because it is compatible with tert-butylation reactions, has a high propensity to form solid product, allows efficient $N$-alkylation under mild conditions and could be easily and selectively removed during solid phase synthesis [6]. $\mathrm{N}$-Protection of commercially available amino alcohols 1a-f was performed with 2-Nos-Cl under standard conditions followed by $O$-tert-butylation of compounds $\mathbf{2 a - f}$ with magnesium perchlorate and di-tert-butylcarbonate in refluxing dichloromethane (Figure 1) [7].

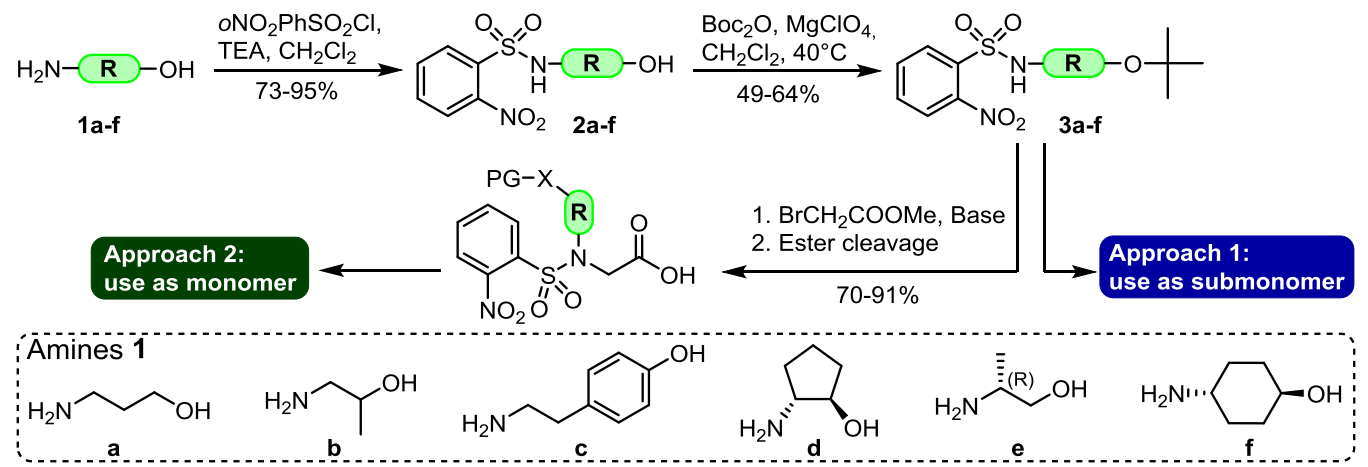

Fig. 1. Synthesis of O-protected N-substituted arylsulfonamides and their use in peptoid synthesis. 

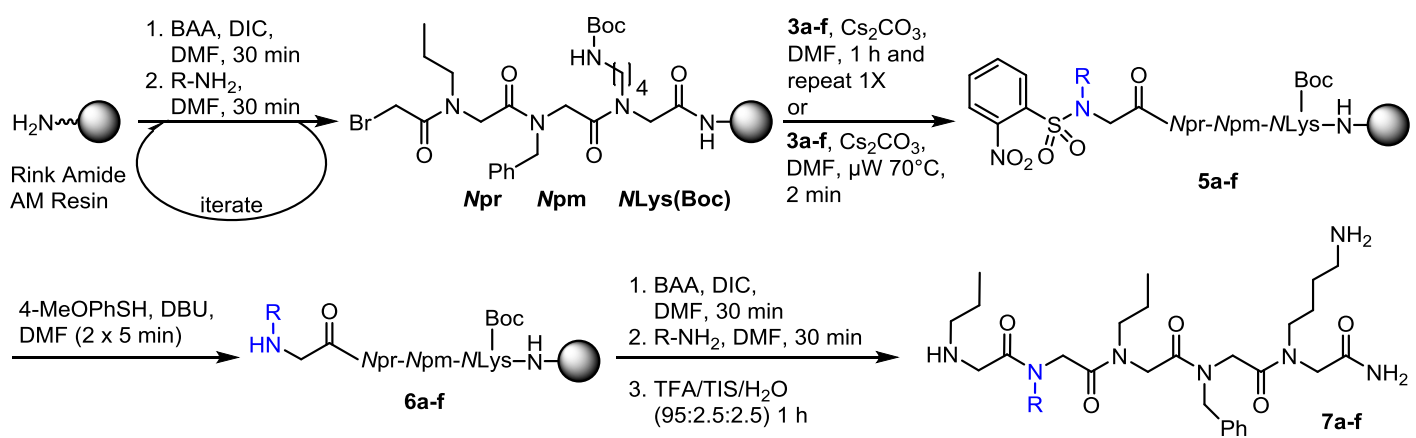

Fig. 2. Synthesis of peptoid oligomers 7 a-f with $N, O$-protected submonomers $3 a-f$.

A first study was performed with the submonomer $N$-(2-methoxyethyl)-2-nitrobenzenesulfonamide on a supported bromoacetylated Phe to identify the optimal conditions for bromine displacement with the $N$-substituted arylsulfonamide derivatives [7]. Different reaction parameters were tested and after the nucleophilic displacement and cleavage from the Rink amide resin, the conversion rate was evaluated by HPLC. In our hands the reaction proceeded poorly in DMSO in the presence of $\mathrm{K}_{2} \mathrm{CO}_{3}$ with or without microwave $(\mu \mathrm{W})$ irradiation and the use of 1,8-diazabicyclo[5.4.0]undec-7-ene (DBU) as base yielded a mixture of compounds. The use of DMF as solvent increased the conversion rate and an improvement was observed when the quantities of submonomer and $\mathrm{K}_{2} \mathrm{CO}_{3}$ were doubled. Under $\mu \mathrm{W}$ irradiation, a higher temperature did not improve the conversion rate but its combination with a longer reaction time increased the conversion to $81 \%$. The best results have been obtained when $\mathrm{K}_{2} \mathrm{CO}_{3}$ was replaced by $\mathrm{Cs}_{2} \mathrm{CO}_{3}$. By using $\mathrm{Cs}_{2} \mathrm{CO}_{3}$ as base, the conversion rates were significantly increased and allowed the reduction of submonomer equivalents necessary to reach completion when $\mu \mathrm{W}$ irradiation was applied. Next, the best room temperature and $\mu \mathrm{W}$ conditions were applied to the supported bromoacetylated Phe with the $N, O$-protected submonomers 3a-f. The results showed that the reaction was complete with submonomers 3a-c but did not reach completion with more hindered derivatives 3d-f. To overcome this drawback and assure complete displacement, the quantity of submonomers was increased to 12 equiv and $\mathrm{Cs}_{2} \mathrm{CO}_{3}$ to 18 equiv. In this case, complete conversion was observed with compounds 3d-f for $\mu \mathrm{W}$ conditions while at room temperature the step had to be repeated once to reach comparable conversion rates. To demonstrate the compatibility of building blocks 3a-f with the submonomer method, peptoid heteropentamers based on the sequence $N$ pr- $N$ xxx- $N$ pr- $N$ pm- $N$ lys were prepared on solid support (Figure 2). Following cleavage from the resin, the crude purities of oligomers 7a-f were evaluated by HPLC and the isolated yields calculated after purification (Table 1). The results showed that $N$-substituted arylsulfonamide derivatives can be efficiently used in peptoid synthesis by the submonomer method.

Table 1. Crude purities and isolated yields for peptoid oligomers containing amino alcohols 1a-f.

\begin{tabular}{|c|c|c|c|c|c|c|c|}
\hline & \multirow[t]{2}{*}{ Peptoid } & \multicolumn{2}{|c|}{ Room temp } & \multicolumn{2}{|c|}{ Microwave } & \multicolumn{2}{|c|}{ Monomer } \\
\hline & & $\begin{array}{c}\text { Purity }^{a} \\
(\%)\end{array}$ & $\begin{array}{c}\text { Yield }^{b} \\
(\%)\end{array}$ & $\begin{array}{c}\text { Purity }^{a} \\
(\%)\end{array}$ & $\begin{array}{c}\text { Yield }^{b} \\
(\%)\end{array}$ & $\begin{array}{c}\text { Purity }^{a} \\
(\%)\end{array}$ & $\begin{array}{c}\text { Yield }^{b} \\
(\%)\end{array}$ \\
\hline $7 \mathbf{a}$ & $N$ pr-1a- $N$ pr- $N$ pm-Nlys & 91 & 58 & 90 & 67 & 92 & 59 \\
\hline $7 b$ & $N$ pr-1b-Npr-Npm-Nlys & 88 & 58 & 85 & 66 & 88 & 68 \\
\hline $7 c$ & $N$ pr-1c-Npr-Npm-Nlys & 97 & 76 & 96 & 76 & 89 & 71 \\
\hline 7d & $N$ pr-1d-Npr-Npm-Nlys & 39 & 56 & 54 & 68 & 58 & 38 \\
\hline $7 e$ & $N$ pr-1e- $N$ pr- $N$ pm- $N$ Lys & 71 & 71 & 88 & 79 & & \\
\hline $7 f$ & $N$ pr-1f- $-N p r-N$ pm- $N$ lys & 50 & 48 & 63 & 67 & & \\
\hline $7 g$ & $N$ pr-1c- $N$ pr-1c- $N$ lys & 76 & 55 & 93 & 67 & & \\
\hline
\end{tabular}

${ }^{a}$ Crude purities were determined by integrating the peaks in the HPLC trace $(\lambda=220 \mathrm{~nm}) ;{ }^{b}$ Isolated yield after purification by HPLC and lyophilization. Based on initial loading of Rink Amide AM resin (0.56 mmol/g). 


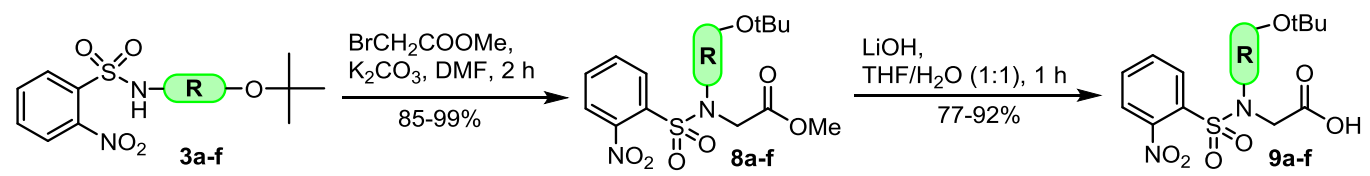

Fig. 3. Synthesis of $N$-arylsulfonyl-N-substituted glycines $\mathbf{9 a - f}$.

In a second study, the $N$-arylsulfonamide building blocks 3a-f were used to prepare protected $\mathrm{N}$-substituted glycine monomers 9a-f (Figure 3). The monomer approach could be more appropriate for hindered $\alpha$-disubstituted amino alcohols. The first study with submonomers 3a-f showed that the bromine displacement on solid support was the limiting step. In the monomer approach [8], this step is performed in solution and could be more efficient since a greater quantity of alkylating derivatives can be used. This strategy offers, for more hindered residue, an important submonomer economy and the opportunity to increase oligomer purities and isolated yields. Different approaches were evaluated to prepare monomers 9a-f and the most efficient was found to be $N$-alkylation with methyl bromoacetate and $\mathrm{K}_{2} \mathrm{CO}_{3}$ in DMF followed by selective ester cleavage with LiOH (Figure 3). After purification by flash chromatography, the $N$-2-Nos- $N$-substituted glycines 9a-f were obtained in good yields (70-91\% for 2 steps). To demonstrate their compatibility with peptoid synthesis, monomers 9a-d ( 3 equiv) were coupled to the peptoid $N$ pr- $N$ pm- $N$ Lys on solid support using HATU ( 3 equiv) as coupling reagent in presence of NMM (6 equiv) in DMF for $3 \mathrm{~h}$ to yield 5a-d. Afterward, the 2-Nos was cleaved with a solution of $p$-methoxybenzenethiol and DBU and a last residue was added to peptoids 6a-f by standard submonomer methodology as described above (Figure 2). After cleavage from the resin, the crude purities of oligomers 7a-d were evaluated by HPLC (Table 1). The results showed that $N$-substituted arylsulfonamides are convenient building blocks to prepare $N$-substituted glycine monomers that can be efficiently used in the synthesis of peptoids or $N$-alkylated peptides on solid support.

Overall, these results demonstrate that $N$-substituted 2-nitrobenzenesulfonamides can be used in peptoid synthesis as submonomers or monomers. In the submonomer approach, $\mu \mathrm{W}$ irradiation is recommended for $\alpha$-substituted amines as previously observed. We have also shown that the more efficient conditions with $\mathrm{Cs}_{2} \mathrm{CO}_{3}$ are compatible with the submonomer method and could be used to introduce expensive or commercially unavailable side chain protected amines and relevant functional groups. Moreover, $N$-substituted arylsulfonamides are also useful building blocks to prepare side chain protected $N$-substituted glycines for the monomer approach. The conditions used in this work allow a substantial building block economy compared to standard conditions. Simple and affordable, the described procedures represent a complementary and interesting alternative approach to introduce relevant functionalized side chains into peptoids and peptide-peptoid hybrids, increasing the chemical diversity accessible with both submonomer and monomer approaches.

\section{Acknowledgments}

Simon Vézina-Dawod and Steve Jobin thank the Fond d'enseignement et de recherche de la Faculté de pharmacie de l'Université Laval (FER) for research scholarships. This work was supported by the National Sciences and Engineering Research Council of Canada (NSERC) (371503-2010).

\section{References}

1. Fowler, S.A., Blackwell, H.E. Org. Biomol. Chem. 7, 1508-1524 (2009), http://dx.doi.org/10.1039/B817980H

2. Dohm, M.T., Kapoor, R., Barron, A.E. Curr. Pharm. Des. 17, 2732-2747 (2011), http://dx.doi.org/10.2174/138161211797416066

3. Miller, S.M., Simon, R.J., Ng, S., Zuckermann, R.N., Kerr, J.M., Moos W.H. Bioorg. Med. Chem. Lett. 4, 2657-2662 (1994), http://dx.doi.org/10.1016/S0960-894X(01)80691-0

4. Kwon, Y.U., Kodadek, T. Chem. Biol. 14, 671-677 (2007), http://dx.doi.org/10.1016/j.chembiol.2007.05.006

5. Zuckermann, R.N., et al. J. Am. Chem. Soc. 114, 10646-10647 (1992), http://dx.doi.org/10.1021/ja00052a076

6. Fukuyama, T., Jow, C.K., Cheung, M. Tetrahedron Lett. 36, 6373-6374 (1995), http://dx.doi.org/10.1016/0040-4039(95)01316-A

7. Vézina-Dawod, S., Derson, A., Biron, E. Tetrahedron Lett. 56, 382-385 (2015), http://dx.doi.org/10.1016/j.tetlet.2014.11.104

8. Kruijtzer, J.A.W., et al. Chem. Eur. J. 4, 1570-1580 (1998), http://dx.doi.org/10.1002/(SICI)15213765(19980807)4:8<1570::AID-CHEM1570>3.0.CO;2-2 